\title{
Primary Prevention of Fatal Ventricular Arrhythmias With Implantable Cardioverter- Defibrillator Therapy - An Analysis of Implications Based on MADIT II Criteria
}

\author{
Zia I Carrim*, Ashraf A Khan, Shazad Aslam
}

\begin{abstract}
Aims: The primary aim of this retrospective study was to determine the proportion of patients with myocardial infarction (MI) who fulfil the criteria of the Multicenter Automatic Defibrillator Implantation Trial (MADIT) II and the implications of MADIT II criteria in practice. Methods: We performed a retrospective analysis of three hundred and ninety four admissions to the Coronary Care Unit (CCU) of the Royal Infirmary of Edinburgh. We selected those with myocardial infarction (MI) and attempted to retrieve electronic copies of their echocardiogram reports. When available, these were used to assess requirement for primary-prevention Implantable Cardioverter Defibrillator (ICD) therapy based on reported left ventricular function. Results: One hundred and ninety patients were admitted to the $\mathrm{CCU}$ with a diagnosis of MI. Of these, 100 patients $(51.5 \%)$ had an echocardiogram. Requirement for ICD therapy was unlikely in $87(87 \%)$, probable in $6(6 \%)$ and necessary in $7(7 \%)$. Since a significant number of patients in the probable category were also likely to meet MADIT II criteria, we concluded that the proportion of patients requiring primary-prevention ICD therapy would be no less than $7 \%$ and more likely to be $13 \%$. Conclusion: In the context of a busy teaching hospital, a figure of $13 \%$ for the requirement of ICD therapy in MI patients represents annual implantation activity of at least 100 per million. This is likely to have very significant resource implications.
\end{abstract}

\section{INTRODUCTION}

Structural abnormalities of the coronary arteries and their consequences are known to be responsible for $80 \%$ of fatal arrhythmias $(1,2)$. Huikuri et al. have shown that patients with previous myocardial infarction, low ejection fraction and ventricular tachycardia are at much higher risk of sudden cardiac death (SCD) compared to the general population (3). Several trials have investigated primary and secondary prevention of

*To whom correspondence should be addressed: Zia I Carrim MB $\mathrm{ChB}$ (Hons), Formerly Cardiology Resident, Royal Infirmary of Edinburgh. Flat 3, 19 High Riggs, Edinburgh, EH3 9BW, Scotland, United Kingdom.

email: zia.carrim@doctors.org.uk
SCD using anti-arrhythmic drugs or implantable cardioverter-defibrillators (ICDs). Primary-prevention trials based on anti-arrhythmic drugs, other than betablockers, have demonstrated either no survival benefit $(4,5)$ or, at worst, adverse outcomes (6.7). In contrast, recent evidence from the Multicenter Automatic Defibrillator Trial II (MADIT II) suggests that prophylactic ICD therapy produces a significant survival benefit in patients with prior myocardial infarction and advanced left ventricular dysfunction (8).

This finding is consistent with earlier trials, such as MADIT, the Multicenter Unsustained Tachycardia Trial (MUSTT) and the Antiarrhythmics Versus Implantable Defibrillators study (AVID), which showed a 
significant benefit of ICD therapy over anti-arrhythmic medication $(9,10)$. Compared to the design of MADIT and MUSTT, where patients underwent invasive electrophysiological testing to determine risk of arrhythmia, MADIT II criteria were simplified on the grounds that myocardium damaged by infarction and rendered severely dysfunctional is a trigger for fatal arrhythmias by the process of macro re-entry (8). Therefore, the findings of MADIT II apply to a large proportion of patients with previous MI and severely impaired left ventricular function.

A major obstacle to the application of findings from randomised trials, such as MADIT II, is the absence of data on the proportion of patients who fulfil inclusion criteria. The purpose of our audit was to address this problem and to evaluate, both quantitatively and qualitatively, the potential implications of MADIT II criteria in the context of a busy teaching hospital.

\section{METHODS}

Using the Ward Register, we identified all admissions to the Coronary Care Unit (CCU) at the Royal Infirmary of Edinburgh over a period of 6 months from 1st October 2001 to 31st March 2002. Of these, we selected those with a diagnosis of myocardial infarction (MI) based on ST segment elevation and a rise in cardiac enzymes. We then attempted to retrieve electronic copies of echocardiogram reports for each patient. A retrieval rate of $100 \%$ was not expected on account of the absence of electronic reports for patients having had portable echocardiography performed in the CCU. In cases where reports were available, only the latest was considered. Patients were then classified according to their reported left ventricular function.

MADIT II findings apply to patients with prior MI and a left ventricular ejection fraction (LVEF) less than $30 \%$ (8). While the design of this simple retrospective study did not permit us to quantify LVEF, we reasoned that primary-prevention ICD therapy in our study population could be classified as necessary, probable or unlikely on the basis of the echocardiogram report which provided a subjective assessment of left ventricular function. Patients with reported normal, mild or mild / moderate impairment were considered unlikely to require ICD-therapy, those with moderate or moderate/severe impairment were probable candidates, and those with severe impairment were necessary candidates.

In determining the proportion of MI patients who would require primary-prevention ICD therapy, two additional factors were considered. First, amongst patients who were probable candidates for ICD therapy, it was likely that a significant proportion would meet MADIT II criteria if left ventricular function were formally assessed. Secondly, it is the policy of our CCU to refer for departmental echocardiography patients who do not have clinical evidence of left ventricular impairment in the immediate post-MI period. This implies that amongst patients who did not have a departmental echocardiogram, the proportion requiring ICD therapy would be greater than amongst those who did have an echocardiogram. The sum of the proportion of patients classified as necessary and probable candidates was therefore deemed a better estimate of the proportion of patients requiring ICD therapy $\left(\mathrm{p}_{\mathrm{ICD}}\right)$.

Using mortality data from MADIT II and our findings, we constructed a mathematical model to forecast the number of patients requiring follow-up after ICD therapy for primary-prevention. This model, based on a geometric series, assumes constant annual ICD implantation activity and mortality such that the total burden of ICD patients at the end of each year can be expressed as follows:

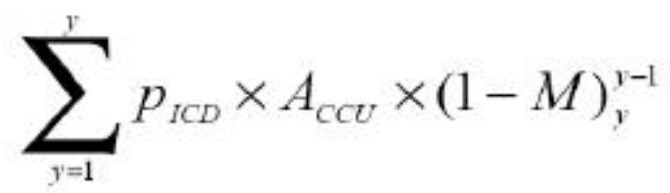

$\left(\mathrm{y}=\right.$ number of years, $\mathrm{p}_{\mathrm{ICD}}=$ proportion requiring $\mathrm{ICD}$ therapy, $\mathrm{A}_{\mathrm{CCU}}=$ total $\mathrm{MI}$ admissions to $\mathrm{CCU} /$ year, $\mathrm{M}=$ population mortality)

Our projections were limited to 5 years because of the potential impact of other variables, such as lifetime of the ICD device, which necessitate consideration in a long-term evaluation.

\section{RESULTS}

Table I summarises our findings. Between 1st October 2001 and 31st March 2002, a total of 394 patients were admitted to the CCU of our teaching hospital. Of these, $190(48.2 \%)$ had a clear diagnosis of MI. $131(68.9 \%)$ were males and $59(31.1 \%)$ were females. The age range was 30 to 95 years (Mean 65.7 years). We retrieved a total of 100 echocardiogram (Echo) reports, representing $51.5 \%$ of the total number. Using our classification, requirement for ICD therapy was unlikely in 87 patients $(87 \%)$, probable in $6(6 \%)$ and necessary in 7 (7\%). By extrapolation, these results suggest that, over a 12-month period, requirement for ICD therapy will be probable in 23 and necessary in 27 (figure 1). We concluded that our overall annual estimate for new ICD implants would be at least 27 (7\% of CCU MI admissions being necessary candidates) but more likely to be closer to 50 (13\% of CCU MI admissions). This represents a potential increase of $125 \%$ in ICD implantation activity in our hospital (table I). 
Table I - Summary of findings for each calendar month (October 2001 to March 2002)

\begin{tabular}{|c|c|c|c|c|c|c|c|}
\hline & Oct & Nov & Dec & Jan & Feb & Mar & Overall \\
\hline Total Admissions & 68 & 53 & 65 & 78 & 57 & 73 & 394 \\
\hline MI Adminssions (\%) & $34(50.0)$ & $15(28.3)$ & $29(44.6)$ & $45(57.7)$ & $34(59.6)$ & $33(45.2)$ & $190(48.2)$ \\
\hline Mean age / years & 66.6 & 66.7 & 65.0 & 67.6 & 65.7 & 62.6 & 65.7 \\
\hline No. of Males with MI (\%) & $21(61.8)$ & $13(86.7)$ & $21(72.4)$ & $30(66.7)$ & $22(64.7)$ & $24(72.7)$ & $131(68.9)$ \\
\hline No. of Females with MI (\%) & $13(38.2)$ & $2(13.3)$ & $8(27.6)$ & $15(33.3)$ & $12(35.3)$ & $9(27.3)$ & $59(31.1)$ \\
\hline Retrieved Echos (\%) & $25(73.5)$ & $9(60.0)$ & $11(37.9)$ & $23(51.1)$ & $16(47.1)$ & $16(48.5)$ & $100(51.5)$ \\
\hline \multicolumn{8}{|l|}{ ICD Requirement: } \\
\hline Unlikely & 21 & 8 & 10 & 19 & 15 & 14 & 87 \\
\hline Probable & 2 & 1 & 0 & 2 & 0 & 1 & 6 \\
\hline Required & 2 & 0 & 1 & 2 & 1 & 1 & 7 \\
\hline $2^{\circ}$ prevention ICDs & 8 & 3 & 3 & 1 & 3 & 2 & 20 \\
\hline
\end{tabular}

$2^{\circ}$ prevention ICDs $=$ Seconday prevention ICD implants

\section{Projected ICD implants over 12 months}

$\square$ Necessary $\mathrm{EProbable} \square$ Unlikely

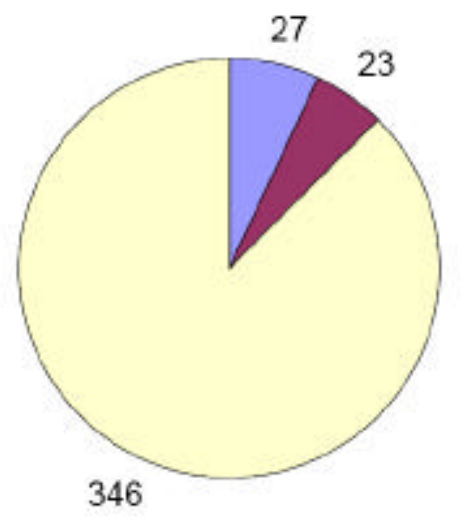

Using MADIT II findings, we calculated an annual mortality rate of $9.1 \%$ for patients having received ICD therapy for primary prevention (8). We then applied our model to forecast the number of patients requiring follow-up for primary prevention ICD therapy in our hospital:

$$
\sum_{y=1}^{y} 0.13 \times 380 \times(1-0.091)_{y}^{y-1}
$$

$\left(\mathrm{y}=\right.$ number of years, $\mathrm{p}_{\mathrm{ICD}}=$ proportion requiring $\mathrm{ICD}$ therapy $=0.13, \mathrm{~A}_{\mathrm{CCU}}=$ total $\mathrm{MI}$ admissions to $\mathrm{CCU} /$ year $=380, \mathrm{M}=$ population mortality $=0.091$ )

Our results, illustrated in figure 2, show that the number of primary-prevention ICD patients requiring follow-up is likely to reach 100 within 2 years and then double within 5 years of implementing MADIT II criteria.

\section{DISCUSSION}

It is estimated that secondary-prevention ICD implantation averages 17 per million in the United Kingdom. This figure has been reported as being less than half of that for Western Europe and less than $10 \%$ of that in the USA 12. Prior to MADIT II, the use of ICD therapy for primary prevention was controversial. Studies such as the Canadian Implantable Defibrillator Study (CIDS) and Coronary Artery Bypass Graft (CABG) Patch trial found no statistically significant reduction in mortality from the use of ICD therapy for secondary prevention and primary prevention, respectively $(13,14)$. The 2002 guidelines from the American College of Cardiology and the American Heart Association (ACC/AHA) include the use of ICD therapy for primary prevention in patients with left ventricular ejection fraction of less than or equal to $30 \%$ at least 1 month post myocardial infarction and 3 months post coronary artery revascularization surgery (15). This is currently a class IIa recommendation based on increasing evidence in favour of ICD therapy in this setting. Implementation of this recommendation in conjunction with MADIT II criteria is likely to have important implications for resource allocation and

\section{Projected cumulative increase in primary- prevention ICD patients}

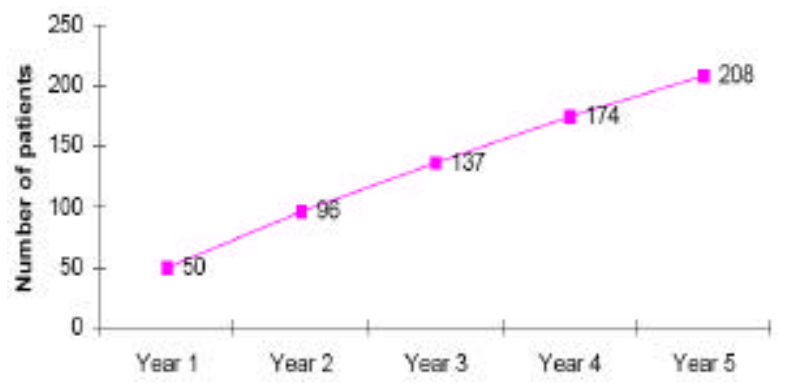

Figure 2 - Cumulative burden of primary-prevention ICD patients over 5 years. 
policy making in the National Health Service (NHS). This discussion focuses on the ramifications in the context of a busy teaching hospital and specialist regional implanting centre.

In the MADIT II trial, patients who were eligible for inclusion had undergone objective assessment of LVEF by angiography, radionuclide scanning or echocardiography (12). In our audit study, only $51.5 \%$ of patients were referred for echocardiography. There was also no formal objective assessment of LVEF. While these constitute intrinsic methodological limitations, they are also a reflection of current practice. Evidence-based selection and exclusion of patients for primary-prevention ICD therapy is likely to involve a much greater proportion of $\mathrm{CCU}$ patients undergoing formal assessment of LVEF. Since echocardiography is readily available and relatively cheap, expansion of the service may be warranted in centres implementing MADIT II findings.

As suggested by our study, about $13 \%$ of patients admitted to CCU with MI require primary-prevention ICD therapy. On an annual basis, the estimated 50 new cases for a population of 500000 in Edinburgh (Scotland) are likely to increase ICD implantation activity by at least $125 \%$. Providing for a surge in demand of this magnitude will have implications for theatre time, staffing levels and the provision of extra funds for hardware. Whereas implantation activity is unlikely to vary significantly in the short term, the cumulative burden of primary-prevention ICD patients requiring follow-up is likely to increase substantially (figure 2). According to guidelines from the British Pacing and Electrophysiology Group (BPEG), every increment of 100 patients justifies an additional followup clinic run by 2 technicians and 1 physician (16). Based on our model, by the end of year 5, 2 follow-up clinics would be running at capacity.

Our evaluation of the implications of MADIT II criteria has been limited to a 5-year period on account of the potential influence, in the long-term, of several factors. As implantation activity increases, the cost of ICD devices is expected to fall as a result of economies of scale. Improvements in technology are likely to improve the lifetime of the ICD device, thereby reducing the requirement for replacement. As primaryprevention ICD therapy gains acceptance, more District General Hospitals may be involved in ICD implantation and follow-up. This can reduce the burden on specialist centres but also involves more emphasis on, and investment in, the training of cardiologists with an interest in pacemakers and ICDs. Newer approaches such as ablative therapy may also influence the use of ICD therapy. Although no trials have been carried out, there have been reports of recurrent monomorphic VT following MI being treated successfully with single catheter ablation and concomitant administration of anti-arrhythmic drugs (17).

In conclusion, our retrospective study shows that at least $13 \%$ of $\mathrm{MI}$ admissions to $\mathrm{CCU}$ are eligible for primary-prevention ICD therapy. In the context of a busy teaching hospital, annual implantation activity, using MADIT II criteria, can be expected to reach as much as 100 per million of population. As suggested by our model, the cumulative number of patients having received primary prevention ICD therapy is likely to have major implications for follow-up clinics. To our knowledge, this is the first study investigating the implications of MADIT II criteria. While this study provides useful preliminary information, further studies are needed for more precise evaluation of short and long-term cost implications of primary-pevention ICD therapy.

\section{ACKNOWLEDGEMENTS}

The authors would like to thank Dr Neil R Grubb, Consultant Cardiologist, who provided valuable feedback on an early draft of this paper. No funding was required for this study.

\section{REFERENCES}

1. Myerburg RJ, Interian A, Mitrani RM, Kessler KM, Castellanos A. Frequency of sudden cardiac death and profiles of risk. Am J Cardiol 1997;80: 10F-19F

2. Myerburg RJ, Kessler KM, Castellanos A. Sudden cardiac death: Structure, function, and time-dependence of risk. Circulation 1992; 85(suppl I): I2 - I10

3. Huikuri HV, Castellanos A, Myerburg RJ. Sudden death due to cardiac arrhythmias. N Eng J Med 2001; 345: 1473 -82.

4. Julian DG, Camm AJ, Frangin G, et al. Randomised trial of effect of amiodarone on mortality in patients with leftventricular dysfunction after recent myocardial infarction: EMIAT. Lancet 1997; 349: 667-74.

5. Cairns JA, Connolly SJ, Roberts R, Gent M. Randomised trial of outcome after myocardial infarction in patients with frequent or repetitive ventricular premature depolarisations: CAMIAT. Lancet 1997; 349: 675-82.

6. Echt DS, Liebson PR, Mitchell LB, and the CAST Investigators. Mortality and morbidity in patients receiving encainide, flecainide, or placebo: the Cardiac Arrhythmia Suppression Trial. N Eng J Med 1991; 324: 781-8.

7. Waldo AL, Camm AJ, deRuyter H, et al. Effect of d-sotalol on mortality and morbidity in patients with left ventricular dysfunction after recent and remote myocardial infarction. Lancet 1996; 348: 7-12.

8. Moss AJ, Zareba W, Hall WJ, et al. Prophylactic implantation of a defibrillator in patients with myocardial infarction and reduced ejection fraction: Multicenter Automatic Defibrillator Implantation Trial II. N Eng J Med 2002; 346: 877-883.

9. Moss AJ, Hall WJ, Cannom DS, et al. Improved survival with an implanted defibrillator in patients with coronary disease at high risk for ventricular arrhythmia : Multicenter Automatic Defibrillator Implantation Trial. N Eng J Med 1996; 335: 193340.

10. Buxton AE, Lee KL, Fisher JD, Josephson ME, Prystowsky EN, 
Hafley GA. A randomized study of the prevention of sudden death in patients with coronary artery disease: Multicenter Unsustained Tachycardia Trial. N Eng J Med 1999; 341:188290.

11. The Antiarrhythmics versus Implantable Defibrillators (AVID) Investigators. A comparison of anti-arrhythmic therapy with implantable defibrillators in patients resuscitated from near-fatal ventricular arrhythmias. N Eng J Med 1997; 337:1576 - 83.

12. Cuser JP, Connelly DT. Implantable defibrillators for life threatening ventricular arrhythmias are more effective than antiarrhythmic drugs in selected high risk patients. Br Med J 1998; 317:762-3.

13. Connolly SJ, Gent M, Roberts RS et al. Canadian implantable defibrillator study (CIDS): a randomized trial of the implantable cardioverter defibrillator against amiodarone. Circulation 2000; 101: 1297-302.

14. Bigger JT Jr. Prophylactic use of implanted cardiac defibrillators in patients at high risk for ventricular arrhythmias after coronary-artery bypass graft surgery. Coronary Artery Bypass Graft (CABG) Patch Trial Investigators. N Engl J Med 1997; 337: 1569-75.

15. Gregoratos G, Abrams J, Epstein A, et al. ACC/AHA/NASPE 2002 Guideline update for implantation of ca for implantation of cardiac pacemakers and antiarrhythmia devices: Summary article: A report of the American College of Cardiology/American Heart Assoication Task Force on Practice Guidelines (ACC/AHA/NASPE Committee to update the 1998 Pacemaker Guidelines). Circulation 2002; 106: 2145 - 61.

16. British Pacing and Electrophysiology Group. Guidelines for pacemaker follow up. Heart 1996; 76: 458-60.

17. O'Donnell D, Bourke J, AnilKumar R, Simoneoidou E, Furniss S. Radiofrequency ablation for post-infarction ventricular tachycardia. Report of a single centre experience of 112 cases. Eur Heart J 2002; 23: 1699- 705.amison D, Creese A and Prentice T. The world health report 1999: making a difference. France: World Health Organization, 1999.

Z Carrim was formerly a resident in Cardiology at the Royal Infirmary in Edinburgh. He is now a Senior House Officer in Ophthalmology at the Southern General Hospital in Glasgow.

A Khan is a final year medical student at Edinburgh University. He has an interest in Cardiology but would like to work for a humanitarian organization such as Medecins Sans Frontiere before launching himself into this.

S Aslam is currently a Senior House Officer in Medicine at the Manchester Royal Infirmary. He intends to pursue a career in Radiology. 